\title{
Matrix Form of Deriving High Order Schemes for the First Derivative
}

\author{
Hassan Abd Salman Al-Dujaly ${ }^{1} \quad$ Yinlin Dong ${ }^{2}$
}

\author{
${ }^{1}$ Mustansiriyah University - College of Science, Baghdad, Iraq , 009647800552540 , \\ hassanaldujaly@uomustansiriyah.edu.iq , https://orcid.org/0000-0001-9771-0129 \\ $2 *$ Corresponding author: Yinlin Dong, ${ }^{2}$ University of Central Arkansas, Conway, Arkansas, USA \\ 0015014505684 / ydong5@uca.edu, https://orcid.org/0000-0003-4030-3383
}

Received 27/10/2019, Accepted 10/3/2020, Published 8/9/2020

This work is licensed under a Creative Commons Attribution 4.0 International License.

\begin{abstract}
:
For many problems in Physics and Computational Fluid Dynamics (CFD), providing an accurate approximation of derivatives is a challenging task. This paper presents a class of high order numerical schemes for approximating the first derivative. These approximations are derived based on solving a special system of equations with some unknown coefficients. The construction method provides numerous types of schemes with different orders of accuracy. The accuracy of each scheme is analyzed by using Fourier analysis, which illustrates the dispersion and dissipation of the scheme. The polynomial technique is used to verify the order of accuracy of the proposed schemes by obtaining the error terms. Dispersion and dissipation errors are calculated and compared to show the features of high order schemes. Furthermore, there is a plan to study the stability and accuracy properties of the present schemes and apply them to standard systems of time dependent partial differential equations in CFD.
\end{abstract}

Key words: Compact Scheme, Dispersion, Dissipation, High Order, Wave Number

\section{Introduction}

Many phenomena in physics like turbulent fluid flows have a range of time scales and space scales. The length scales that are resolved by computation models are determined by the spectral resolution (1), and the accuracy with which these scales are represented depends upon the numerical scheme (2). Fourier analysis of finite difference schemes (3) shows that the errors in computing the derivatives can be quite large for smaller scales. This small scale inaccuracy becomes increasingly important as the energy in the small scales becomes increasingly comparable to that of the large scales, which may result in overwhelming numerical errors in computations such as large eddy simulation of high Reynolds number turbulence (4). Therefore, the numerical schemes should represent all the relevant scales properly.

Finite difference schemes can be classified as explicit and implicit schemes (2). Explicit schemes express the nodal derivatives as an explicit weighted sum of the function values at the adjacent nodes. On the other hand, implicit or compact schemes equate a linear combination of the nodal derivatives to a weighted sum of the function values so that the derivatives must be calculated by solving for a matrix system implicitly. It is well known (4-6) that implicit schemes are significantly more accurate for the small scales than explicit schemes with the same stencil width, i.e., the number of nodes in the scheme. Many implicit or spectral-like compact schemes have been developed (4, 7-9). Since the spectral methods require the flow to be simple in both domain and boundary condition, many attempts have been made to overcome this drawback of the spectral methods (9-10).

In this paper, finite difference approximations of the first derivative will be derived for a discrete data set. In reference (3), such approximations are performed by implicit schemes in which the maximum stencil sizes on the left-hand-side and right-hand-side of the equations are 5 and 7 respectively. In this work, a generalized framework that can handle any stencil size on both sides will be derived, and a matrix form of the approximation will be built, so it is easy to obtain the coefficients in the scheme by solving a linear system. A variety of high order 
schemes will be presented with dispersion and dissipation error analysis.

\section{The derivation of the schemes using the matrix form}

In deriving the finite difference schemes, an approximation of the derivative $f^{\prime}$ can be expressed as a linear combination of $\left\{f_{k-i}, f_{k-i+1}, \ldots, f_{k-1}, f_{k}, f_{k+1}, \ldots, f_{k+j-1}, f_{k+j}\right\}$. A uniform space with mesh size $h$ is considered. The approximation of the first derivative $f_{k}^{\prime}$ can be expressed as follows:

$$
\begin{gathered}
c_{0} f_{k-i}^{\prime}+c_{1} f_{k-i+1}^{\prime}+\cdots+c_{i-2} f_{k-2}^{\prime}+c_{i-1} f_{k-1}^{\prime}+f_{k}^{\prime}+ \\
c_{i+1} f_{k+1}^{\prime}+c_{i+2} f_{k+2}^{\prime}+\cdots+c_{n-1} f_{k+j-1}^{\prime}+c_{n} f_{k+j}^{\prime}= \\
\frac{1}{h}\left[d_{0} f_{k-l}+d_{1} f_{k-l+1}+\cdots+d_{i-2} f_{i-2}+d_{i-1} f_{k-1}+\right. \\
d_{i} f_{k}+d_{i+1} f_{k+1}+d_{i+2} f_{k+2}+\cdots+d_{n-1} f_{k+l-1}+ \\
\left.d_{n} f_{k+l}\right] \text { (1) }
\end{gathered}
$$

The traditional approach is using Tayler series expansion to find the relations between the coefficients $c_{0}, \ldots, c_{n}, d_{0}, \ldots, d_{n}$ as in (1). In this work, the derivation is based on the following system:
The elements of the matrices $P$ and $P^{\prime}$ are derived by assuming that

$$
\begin{aligned}
& P= \\
& {\left[\begin{array}{lllllllll}
f_{-l} & \ldots & f_{-2} & f_{-1} & f_{0} & f_{1} & f_{2} & \ldots & f_{l}
\end{array}\right]_{(m+1) \times(2 l+1)}} \\
& \text { and } \\
&
\end{aligned}
$$
where

$$
\begin{gathered}
f_{k}=\left[\begin{array}{c}
1 \\
x_{k} \\
x_{k}{ }^{2} \\
x_{k}{ }^{3} \\
\vdots \\
\vdots \\
x_{k}{ }^{m-1} \\
x_{k}^{m} \\
0 \\
1 \\
2 x_{k} \\
3 x_{k}{ }^{2} \\
\vdots \\
\vdots \\
(m-1) x_{k}{ }^{m-2} \\
m x_{k}^{m-1}
\end{array}\right]_{(m+1) \times 1} \text { and } f^{\prime}{ }_{k}= \\
{\left[\begin{array}{c} 
\\
\end{array}\right.}
\end{gathered}
$$

$$
P^{\prime} C=\frac{1}{h} P d
$$

Letting $x_{0}=0$ and the mesh size $h=1$ gives the system as follows:

$$
P=\left[\begin{array}{ccccccccc}
1 & 1 & \cdots & 1 & 1 & 1 & \cdots & 1 & 1 \\
-l & 1-l & \cdots & -1 & 0 & 1 & \cdots & l-1 & l \\
l^{2} & (1-l)^{2} & \cdots & 1 & 0 & 1 & \cdots & (l-1)^{2} & l^{2} \\
-l^{3} & (1-l)^{3} & \cdots & -1 & 0 & 1 & \cdots & (l-1)^{3} & l^{3} \\
\vdots & \vdots & \cdots & \vdots & \vdots & \vdots & \cdots & \vdots & \vdots \\
\vdots & \vdots & \cdots & \vdots & \vdots & \vdots & \cdots & \vdots & \vdots \\
(-l)^{m-1} & (1-l)^{m-1} & \cdots & (-1)^{m-1} & 0 & 1 & \cdots & (l-1)^{m-1} & l^{m-1} \\
(-l)^{m} & (1-l)^{m} & \cdots & (-1)^{m} & 0 & 1 & \cdots & (l-1)^{m} & l^{m}
\end{array}\right]_{(m+1) \times(2 l+1)}
$$

$$
\begin{aligned}
& P^{\prime}= \\
& {\left[\begin{array}{ccccccccc}
0 & 0 & 0 & 0 & 0 & 0 & 0 & 0 & 0 \\
1 & 1 & \cdots & 1 & 1 & 1 & \cdots & 1 & 1 \\
-2 i & 2(1-i) & \cdots & -2 & 0 & 2 & \cdots & 2(j-1) & 2 j \\
3 i^{2} & 3(1-i)^{2} & \cdots & 3 & 0 & 3 & \cdots & 3(j-1)^{2} & 3 j^{2} \\
\vdots & \vdots & \cdots & \vdots & \vdots & \vdots & \cdots & \vdots & \vdots \\
\vdots & \vdots & \cdots & \vdots & \vdots & \vdots & \cdots & \vdots & \vdots \\
(m-1)(-i)^{m-2} & (m-1)(1-i)^{m-2} & \cdots & (m-1)(-1)^{m-2} & 0 & (m-1) & \cdots & (m-1)(j-1)^{m-2} & (m-1) j^{m-2} \\
m(-i)^{m-1} & m(1-i)^{m-1} & \cdots & m(-1)^{m-1} & 0 & m & \cdots & m(j-1)^{m-1} & m j^{m-1}
\end{array}\right]_{(m+1) \times(n+1)}}
\end{aligned}
$$




$$
c=\left[\begin{array}{c}
c_{0} \\
c_{1} \\
\vdots \\
c_{t-1} \\
1 \\
c_{t+1} \\
\vdots \\
c_{n-1} \\
c_{n}
\end{array}\right]_{(n+1) \times 1} \quad \text { and } d=\left[\begin{array}{c}
\mathrm{d}_{0} \\
\mathrm{~d}_{1} \\
\mathrm{~d}_{2} \\
\mathrm{~d}_{3} \\
\vdots \\
\vdots \\
\vdots \\
\mathrm{d}_{21-1} \\
\mathrm{~d}_{21}
\end{array}\right]_{(2 l+1) \times 1}
$$

where $i$ and $j$ are positive integers with $n=i+j$ and $l=\max \{i, j\}$.

Remarks:

1. All approximations from eq. (2) are called compact because of using derivatives in the lefthand side, and eq. (2) represents the traditional finite difference approximations for the first derivative if $i=j=0$.

2. If $i=j$, the schemes obtained from eq. (2) are central, and the highest order that can be reached is equal to $m=\max \{2 n, 2\}$.

3 . If $i=j+1$, the schemes obtained from eq. (2) are backward, and the highest order that can be reached is equal to $m=2 n+1$.

4. If $j=i+1$, the schemes obtained from eq. (2) are forward, and the highest order that can be reached is equal to $m=2 n+1$.

\section{When $i=j=1$}

In this case $l=1, n=2$ and $m=4$, and from eq. (2), this results in:

$$
\left[\begin{array}{ccc}
0 & 0 & 0 \\
1 & 1 & 1 \\
-2 & 0 & 2 \\
3 & 0 & 3 \\
-4 & 0 & 4
\end{array}\right]\left[\begin{array}{c}
\mathrm{c}_{0} \\
1 \\
\mathrm{c}_{2}
\end{array}\right]=\frac{1}{h}\left[\begin{array}{ccc}
1 & 1 & 1 \\
-1 & 0 & 1 \\
1 & 0 & 1 \\
-1 & 0 & 1 \\
1 & 0 & 1
\end{array}\right]\left[\begin{array}{l}
\mathrm{d}_{0} \\
\mathrm{~d}_{1} \\
\mathrm{~d}_{2}
\end{array}\right]
$$

From eq. (1), the highest scheme of this set is of order 4, and it is central as follows:

CCSO4: $\quad \frac{1}{4} f_{k-1}^{\prime}+f_{k}^{\prime}+\frac{1}{4} f_{k+1}^{\prime}=\frac{1}{h}\left[\frac{-3}{4} f_{k-1}+\right.$ $\left.\frac{3}{4} f_{k+1}\right]$

\section{When $i=2, j=1$}

In this case $l=2, n=3$ and $m=7$, and from eq. (1) and eq. (2), this results in:

$$
\begin{aligned}
& {\left[\begin{array}{cccc}
0 & 0 & 0 & 0 \\
1 & 1 & 1 & 1 \\
-4 & -2 & 0 & 2 \\
12 & 3 & 0 & 3 \\
-32 & -4 & 0 & 4 \\
80 & 5 & 0 & 5 \\
-192 & -6 & 0 & 6 \\
448 & 7 & 0 & 7
\end{array}\right]\left[\begin{array}{c}
c_{0} \\
c_{1} \\
1 \\
c_{3}
\end{array}\right]} \\
& =\frac{1}{h}\left[\begin{array}{ccccc}
1 & 1 & 1 & 1 & 1 \\
-2 & -1 & 0 & 1 & 2 \\
4 & 1 & 0 & 1 & 4 \\
-8 & -1 & 0 & 1 & 8 \\
16 & 1 & 0 & 1 & 16 \\
-32 & -1 & 0 & 1 & 32 \\
64 & 1 & 0 & 1 & 64 \\
-128 & -1 & 0 & 1 & 128
\end{array}\right]\left[\begin{array}{l}
\mathrm{d}_{0} \\
\mathrm{~d}_{1} \\
\mathrm{~d}_{2} \\
\mathrm{~d}_{3} \\
\mathrm{~d}_{4}
\end{array}\right]
\end{aligned}
$$

BCSO7: $\frac{1}{18} f_{k-2}^{\prime}+\frac{2}{3} f_{k-1}^{\prime}+f_{k}^{\prime}+\frac{2}{9} f_{k+1}^{\prime}=$ $\frac{1}{h}\left[\frac{-47}{216} f_{k-2}-\frac{8}{9} f_{k-1}+\frac{1}{2} f_{k}+\frac{16}{27} f_{k+1}+\frac{1}{72} f_{k+2}\right]$

When $i=1, j=2$

In this case $l=2, n=3$ and $m=7$, and from eq. (1) and eq. (2), this results in:

$$
\begin{aligned}
& {\left[\begin{array}{cccc}
0 & 0 & 0 & 0 \\
1 & 1 & 1 & 1 \\
-2 & 0 & 2 & 4 \\
3 & 0 & 3 & 12 \\
-4 & 0 & 4 & 32 \\
5 & 0 & 5 & 80 \\
-6 & 0 & 6 & 192 \\
7 & 0 & 7 & 448
\end{array}\right]\left[\begin{array}{c}
\mathrm{c}_{1} \\
1 \\
\mathrm{c}_{3} \\
\mathrm{c}_{4}
\end{array}\right]} \\
& =\frac{1}{h}\left[\begin{array}{ccccc}
1 & 1 & 1 & 1 & 1 \\
-2 & -1 & 0 & 1 & 2 \\
4 & 1 & 0 & 1 & 4 \\
-8 & -1 & 0 & 1 & 8 \\
16 & 1 & 0 & 1 & 16 \\
-32 & -1 & 0 & 1 & 32 \\
64 & 1 & 0 & 1 & 64 \\
-128 & -1 & 0 & 1 & 128
\end{array}\right]\left[\begin{array}{l}
\mathrm{d}_{0} \\
\mathrm{~d}_{1} \\
\mathrm{~d}_{2} \\
\mathrm{~d}_{3} \\
\mathrm{~d}_{4}
\end{array}\right]
\end{aligned}
$$

FCSO $7: \frac{2}{9} f_{k-1}^{\prime}+f_{k}^{\prime}+\frac{2}{3} f_{k+1}^{\prime}+\frac{1}{18} f_{k+2}^{\prime}=$ $\frac{1}{h}\left[\frac{-1}{72} f_{k-2}-\frac{16}{27} f_{k-1}-\frac{1}{2} f_{k}+\frac{8}{9} f_{k+1}+\frac{47}{216} f_{k+2}\right]$

\section{When $i=2, j=2$}

In this case $l=2, n=4$ and $m=8$, and from eq. (2), this results in: 


$$
\begin{aligned}
& {\left[\begin{array}{ccccc}
0 & 0 & 0 & 0 & 0 \\
1 & 1 & 1 & 1 & 1 \\
-4 & -2 & 0 & 2 & 4 \\
12 & 3 & 0 & 3 & 12 \\
-32 & -4 & 0 & 4 & 32 \\
80 & 5 & 0 & 5 & 80 \\
-192 & -6 & 0 & 6 & 192 \\
448 & 7 & 0 & 7 & 448 \\
-1024 & -8 & 0 & 8 & 1024
\end{array}\right]\left[\begin{array}{l}
\mathrm{c}_{0} \\
\mathrm{c}_{1} \\
1 \\
\mathrm{c}_{3} \\
\mathrm{c}_{4}
\end{array}\right]} \\
& =\frac{1}{h}\left[\begin{array}{ccccc}
1 & 1 & 1 & 1 & 1 \\
-2 & -1 & 0 & 1 & 2 \\
4 & 1 & 0 & 1 & 4 \\
-8 & -1 & 0 & 1 & 8 \\
16 & 1 & 0 & 1 & 16 \\
-32 & -1 & 0 & 1 & 32 \\
64 & 1 & 0 & 1 & 64 \\
-128 & -1 & 0 & 1 & 128 \\
256 & 1 & 0 & 1 & 256
\end{array}\right]\left[\begin{array}{l}
\mathrm{d}_{0} \\
\mathrm{~d}_{1} \\
\mathrm{~d}_{2} \\
\mathrm{~d}_{3} \\
\mathrm{~d}_{4}
\end{array}\right]
\end{aligned}
$$

From eq. (1), the highest scheme of this set is of order 8 , and it is central as follows:

CCSO8: $\quad \frac{1}{36} f_{k-2}^{\prime}+\frac{4}{9} f_{k-1}^{\prime}+f_{k}^{\prime}+\frac{4}{9} f_{k+1}^{\prime}+$ $\frac{1}{36} f_{k+2}^{\prime}=\frac{1}{h}\left[\frac{-25}{216} f_{k-2}-\frac{20}{27} f_{k-1}+\frac{20}{27} f_{k+1}+\right.$ $\left.\frac{25}{216} f_{k+2}\right]$

\section{When $i=3, j=2$}

In this case $l=3, n=5$ and $m=11$, and from eq. (2), this results in:

$$
\left[\begin{array}{cccccc}
0 & 0 & 0 & 0 & 0 & 0 \\
1 & 1 & 1 & 1 & 1 & 1 \\
-6 & -4 & -2 & 0 & 2 & 4 \\
27 & 12 & 3 & 0 & 3 & 12 \\
-108 & -32 & -4 & 0 & 4 & 32 \\
405 & 80 & 5 & 0 & 5 & 80 \\
-1458 & -192 & -6 & 0 & 6 & 192 \\
5103 & 448 & 7 & 0 & 7 & 448 \\
-17496 & -1024 & -8 & 0 & 8 & 1024 \\
59049 & 2304 & 9 & 0 & 9 & 2304 \\
-196830 & -5120 & -10 & 0 & 10 & 5120 \\
649539 & 11264 & 11 & 0 & 11 & 11264
\end{array}\right]\left[\begin{array}{l}
\mathrm{c}_{0} \\
\mathrm{c}_{1} \\
\mathrm{c}_{2} \\
1 \\
\mathrm{c}_{4} \\
\mathrm{c}_{5}
\end{array}\right]=\frac{1}{h}\left[\begin{array}{ccccccc}
1 & 1 & 1 & 1 & 1 & 1 & 1 \\
-3 & -2 & -1 & 0 & 1 & 2 & 3 \\
9 & 4 & 1 & 0 & 1 & 4 & 9 \\
-27 & -8 & -1 & 0 & 1 & 8 & 27 \\
81 & 16 & 1 & 0 & 1 & 16 & 81 \\
-243 & -32 & -1 & 0 & 1 & 32 & 243 \\
729 & 64 & 1 & 0 & 1 & 64 & 729 \\
-2187 & -128 & -1 & 0 & 1 & 128 & 2187 \\
6561 & 256 & 1 & 0 & 1 & 256 & 6561 \\
-19683 & -512 & -1 & 0 & 1 & 512 & 19683 \\
59049 & 1024 & 1 & 0 & 1 & 1024 & 59049 \\
-177147 & -2048 & -1 & 0 & 1 & 2048 & 177147
\end{array}\right]\left[\begin{array}{l}
\mathrm{d}_{0} \\
\mathrm{~d}_{1} \\
\mathrm{~d}_{2} \\
\mathrm{~d}_{3} \\
\mathrm{~d}_{4} \\
\mathrm{~d}_{5} \\
\mathrm{~d}_{6}
\end{array}\right]
$$

From eq. (1), the scheme has the form:

\section{BCSO11:}

$\frac{1}{200} f_{k-3}^{\prime}+\frac{3}{20} f_{k-2}^{\prime}+\frac{3}{4} f_{k-1}^{\prime}+f_{k}^{\prime}+\frac{3}{8} f_{k+1}^{\prime}+$ $\frac{3}{100} f_{k+2}^{\prime}=\frac{1}{h}\left[\frac{-71}{3000} f_{k-3}-\frac{71}{200} f_{k-2}-\frac{11}{16} f_{k-1}+\right.$ $\left.\frac{1}{3} f_{k}+\frac{5}{8} f_{k+1}+\frac{107}{1000} f_{k+2}+\frac{1}{1200} f_{k+3}\right]$

\section{When $i=2, j=3$}

In this case $l=3, n=5$ and $m=11$, and from eq. (2), this results in:

$\left[\begin{array}{cccccc}0 & 0 & 0 & 0 & 0 & 0 \\ 1 & 1 & 1 & 1 & 1 & 1 \\ -4 & -2 & 0 & 2 & 4 & 6 \\ 12 & 3 & 0 & 3 & 12 & 27 \\ -32 & -4 & 0 & 4 & 32 & 108 \\ 80 & 5 & 0 & 5 & 80 & 405 \\ -192 & -6 & 0 & 6 & 192 & 1458 \\ 448 & 7 & 0 & 7 & 448 & 5103 \\ -1024 & -8 & 0 & 8 & 1024 & 17496 \\ 2304 & 9 & 0 & 9 & 2304 & 59049 \\ -5120 & -10 & 0 & 10 & 5120 & 196830 \\ 11264 & 11 & 0 & 11 & 11264 & 649539\end{array}\right]\left[\begin{array}{l}\mathrm{c}_{1} \\ \mathrm{c}_{2} \\ 1 \\ \mathrm{c}_{4} \\ \mathrm{c}_{5} \\ \mathrm{c}_{6}\end{array}\right]=\frac{1}{h}\left[\begin{array}{ccccccc}1 & 1 & 1 & 1 & 1 & 1 & 1 \\ -3 & -2 & -1 & 0 & 1 & 2 & 3 \\ 9 & 4 & 1 & 0 & 1 & 4 & 9 \\ -27 & -8 & -1 & 0 & 1 & 8 & 27 \\ 81 & 16 & 1 & 0 & 1 & 16 & 81 \\ -243 & -32 & -1 & 0 & 1 & 32 & 243 \\ 729 & 64 & 1 & 0 & 1 & 64 & 729 \\ -2187 & -128 & -1 & 0 & 1 & 128 & 2187 \\ 6561 & 256 & 1 & 0 & 1 & 256 & 6561 \\ -19683 & -512 & -1 & 0 & 1 & 512 & 19683 \\ 59049 & 1024 & 1 & 0 & 1 & 1024 & 59049 \\ -177147 & -2048 & -1 & 0 & 1 & 2048 & 177147\end{array}\right]\left[\begin{array}{c}\mathrm{d}_{0} \\ \mathrm{~d}_{1} \\ \mathrm{~d}_{2} \\ \mathrm{~d}_{3} \\ \mathrm{~d}_{4} \\ \mathrm{~d}_{5} \\ \mathrm{~d}_{6}\end{array}\right]$


From eq. (1), the scheme has the form:

FCSO11:

$$
\begin{aligned}
& \frac{3}{100} f_{k-2}^{\prime}+\frac{3}{8} f_{k-1}^{\prime}+f_{k}^{\prime}+\frac{3}{4} f_{k+1}^{\prime}+\frac{3}{20} f_{k+2}^{\prime}+ \\
& \frac{1}{200} f_{k+3}^{\prime}=\frac{1}{h}\left[\frac{-1}{1200} f_{k-3}-\frac{107}{1000} f_{k-2}-\frac{5}{8} f_{k-1}-\right. \\
& \left.\frac{1}{3} f_{k}+\frac{11}{16} f_{k+1}+\frac{71}{200} f_{k+2}+\frac{71}{3000} f_{k+3}\right]
\end{aligned}
$$

$$
\begin{array}{ccccc}
0 & 0 & 0 & 0 & 0 \\
1 & 1 & 1 & 1 & 1 \\
-6 & -4 & -2 & 0 & 2 \\
27 & 12 & 3 & 0 & 3 \\
-108 & -32 & -4 & 0 & 4 \\
405 & 80 & 5 & 0 & 5 \\
-1458 & -192 & -6 & 0 & 6 \\
5103 & 448 & 7 & 0 & 7 \\
-17496 & -1024 & -8 & 0 & 8 \\
59049 & 2304 & 9 & 0 & 9 \\
-196830 & -5120 & -10 & 0 & 10 \\
649539 & 11264 & 11 & 0 & 11 \\
-2125764 & -24576 & -12 & 0 & 12 \\
1 & 1 \\
-3 & -2 \\
9 & 4 \\
-27 & -8 \\
81 & 16 \\
-243 & -32 \\
729 & 64 \\
-2187 & -128 \\
6561 & 256 \\
-19683 & -512 \\
59049 & 1024 \\
-177147 & -2048 \\
531441 & 4096
\end{array}
$$

From eq. (1), the scheme has the form:

CCSO12:

$\frac{1}{400} f_{k-3}^{\prime}+\frac{9}{100} f_{k-2}^{\prime}+\frac{9}{16} f_{k-1}^{\prime}+f_{k}^{\prime}+\frac{9}{16} f_{k+1}^{\prime}+$ $\frac{9}{100} f_{k+2}^{\prime}+\frac{1}{400} f_{k+3}^{\prime}=\frac{1}{h}\left[\frac{-49}{4000} f_{k-3}-\frac{231}{1000} f_{k-2}-\right.$ $\left.\frac{21}{32} f_{k-1}+\frac{21}{32} f_{k+1}+\frac{231}{1000} f_{k+2}+\frac{49}{4000} f_{k+3}\right]$

\section{Dispersion and dissipation errors from Fourier analysis}

When using finite difference approximations for solving differential equations, the approximate solutions generate errors, which associate with odd and even derivatives terms. These terms contribute to numerical dispersions and dissipations. The numerical solution has a dispersion behavior if the derivative of the leading term is odd while it has a dissipative behavior if the derivative of the leading term is even. In flow
When $i=3, j=3$

In this case $l=3, n=6$ and $m=12$, and from eq. (2), this results in:

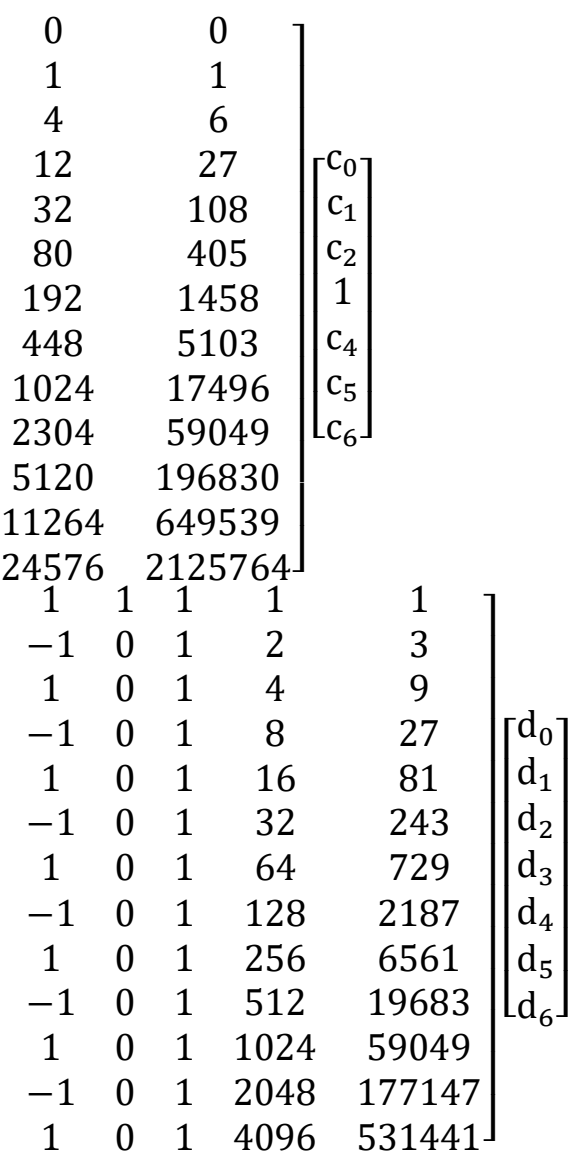

transition problems with small length scales, the behavior of the numerical solutions can be described by dispersion and dissipation errors because the dispersive error terms lead to oscillated solutions while the dissipation error terms affect the smoothing of gradients, see (11) for more details.

The Fourier analysis is an efficient tool of measuring the dispersion and dissipation errors by quantifying the wave number of the numerical approximation (12). The imaginary part and the real part of the wave number introduce the dispersion error and the dissipation error respectively. The wave numbers for the proposed schemes using Fourier analysis are derived, and the dispersion and dissipation errors terms for these schemes are calculated as listed in Tables 1 and 2 below. 
Table 1. Dispersion error terms of the proposed schemes

\begin{tabular}{lc}
\hline Scheme & Dispersion Error Terms \\
\hline CCSO4 & $3 \operatorname{Re}\left[\frac{\sin [k]}{2+\cos [k]}\right]-k$ \\
BCSO7 & $\frac{1}{3} \operatorname{Re}\left[\frac{i(-27+16 \cos [k]+11 \cos [2 k])+5(16+5 \cos [k]) \sin [k]}{16 \cos [k]+\cos [2 k]-i(18 i+8 \sin [k]+\sin [2 k])}\right]-k$ \\
FCSO7 & $\frac{1}{3} \operatorname{Re}\left[\frac{4 i(19+11 \cos [k]) \sin \left[\frac{k}{2}\right]^{2}+5(16+5 \cos [k]) \sin [k]}{18+16 \cos [k]+\cos [2 k]+2 i(4+\cos [k]) \sin [k]}\right]-k$ \\
CCSO8 & $\frac{5}{6} \operatorname{Re}\left[\frac{32 \sin [k]+5 \sin [2 k]}{18+16 \cos [k]+\cos [2 k]}\right]-k$ \\
BCSO1 & $-\frac{1}{30} \operatorname{Re}\left[\frac{2 i(16350 \cos [k]+1902 \cos [2 k]+5(3140+\cos [3 k]))+3150 \sin [k]+756 \sin [2 k]}{225 \cos [k]+36 \cos [2 k]+\cos [3 k]-i(200 i+75 \sin [k]+24 \sin [2 k]+\sin [3 k])}\right]-k$ \\
FCSO1 & $\frac{1}{30} \operatorname{Im}\left[\frac{-2000+375 \cos [k]+1488 \cos [2 k]+137 \cos [3 k]+7875 i \sin [k]+2772 i \sin [2 k]+147 i \sin [3 k]}{200+225 \cos [k]+36 \cos [2 k]+\cos [3 k]+75 i \sin [k]+24 i \sin [2 k]+i \sin [3 k]}\right.$ \\
CCSO1 & $-k$ \\
$\mathbf{2}$ & $\frac{7}{10} \operatorname{Re}\left[\frac{375 \sin [k]+132 \sin [2 k]+7 \sin [3 k]}{200+225 \cos [k]+36 \cos [2 k]+\cos [3 k]}\right]-k$ \\
\hline
\end{tabular}

Table 2. Dissipation error terms of the proposed schemes

\begin{tabular}{cc}
\hline Scheme & Dissipation Error Terms \\
\hline CCSO4 & $3 \operatorname{Im}\left[\frac{\sin [k]}{2+\cos [k]}\right]$ \\
BCSO7 & $\frac{1}{3} \operatorname{Im}\left[\frac{i(-27+16 \cos [k]+11 \cos [2 k])+5(16+5 \cos [k]) \sin [k]}{16 \cos [k]+\cos [2 k]-i(18 i+8 \sin [k]+\sin [2 k])}\right]$ \\
FCSO7 & $-\frac{1}{3} \operatorname{Im}\left[\frac{4 i(19+11 \cos [k]) \sin \left[\frac{k}{2}\right]+5(16+5 \cos [k]) \sin [k]}{18+16 \cos [k]+\cos [2 k]+2 i(4+\cos [k]) \sin [k]}\right]$ \\
CCSO8 & $-\frac{5}{6} \operatorname{Im}\left[\frac{32 \sin [k]+5 \sin [2 k]}{18+16 \cos [k]+\cos [2 k]}\right]$ \\
BCSO11 & $\frac{1}{30}\left(-147+\operatorname{Im}\left[\frac{2 i(15700+16350 \cos [k]+1902 \cos [2 k]+5 \cos [3 k])+3150 \sin [k]+756 \sin [2 k]}{225 \cos [k]+36 \cos [2 k]+\cos [3 k]-i(200 i+75 \sin [k]+24 \sin [2 k]+\sin [3 k])}\right]\right)$ \\
FCSO11 & $\frac{1}{30} \operatorname{Re}\left[\frac{-2000+375 \cos [k]+1488 \cos [2 k]+137 \cos [3 k]+7875 i \sin [k]+2772 i \sin [2 k]+147 i \sin [3 k}{200+225 \cos [k]+36 \cos [2 k]+\cos [3 k]+75 i \sin [k]+24 i \sin [2 k]+i \sin [3 k]}\right.$ \\
CCSO12 & $-\frac{7}{10} \operatorname{Im}\left[\frac{375 \sin [k]+132 \sin [2 k]+7 \sin [3 k]}{200+225 \cos [k]+36 \cos [2 k]+\cos [3 k]}\right]$ \\
\hline
\end{tabular}

Also, the dispersion and dissipation errors of the proposed schemes are compared to show the features of high order schemes as illustrated in Figure 1 and Figure 2 below.

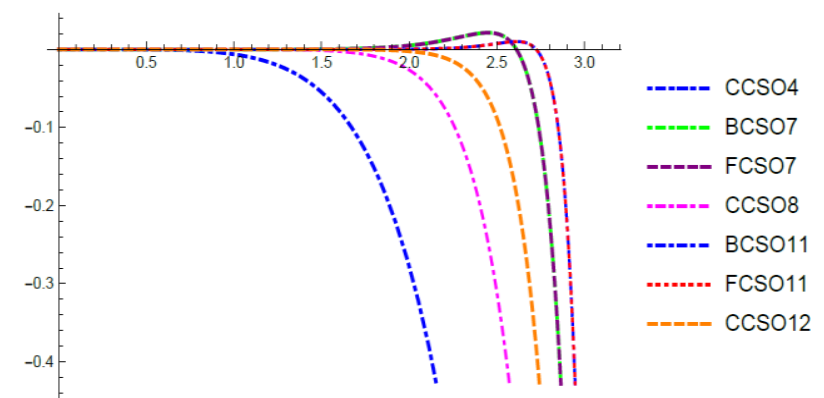

Figure 1. The dispersions errors of the proposed schemes

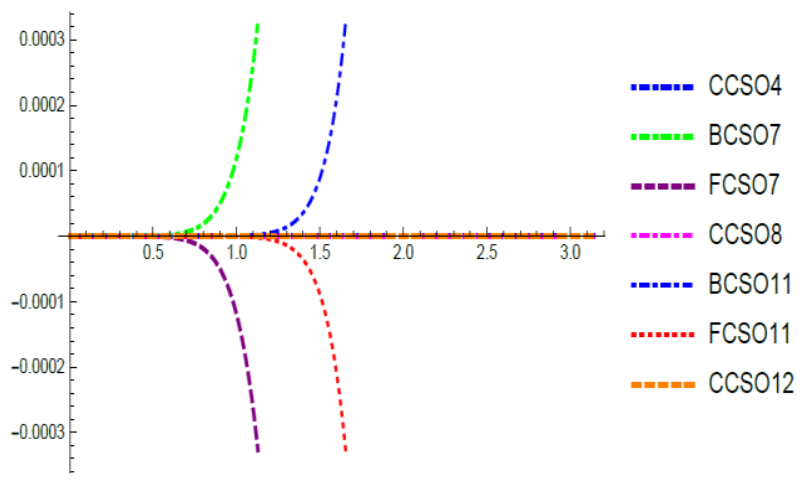

Figure 2. The dispersions errors of the proposed schemes 


\section{Verifying the Order of Accuracy}

To verify the order of accuracy of the proposed schemes, the technique of the $\mathrm{n}^{\text {th }}$ order polynomial $P_{n}(x)=\sum_{k=0}^{n} a_{k} x^{k}$ is used (6), and the first remained coefficients $\left(a_{0}, a_{1}, \ldots, a_{n}\right)$ of this polynomial determine the order of the scheme as indicated in Table 3 below.

Table 3. Error terms of the proposed schemes

\begin{tabular}{ccc}
\hline Schemes & Error terms & Order \\
\hline CCSO4 & $\mathrm{a} 5+2 \mathrm{a} 7+3 \mathrm{a} 9+4 \mathrm{a} 11$ & 4 \\
& $+5 \mathrm{a} 13$ & \\
BCSO7 & $-8(a 8-2 a 9+$ & $10 a 10$ \\
& $-20 a 11$ & 7 \\
& $+67 a 12$ \\
& $-134 a 13)$ & \\
FCSO7 & $8(a 8+2 a 9+10 a 10+20 a 11$ & 7 \\
& $+67 a 12$ \\
& $+134 a 13)$ & \\
CCSO8 & $16(a 9+10 a 11+67 a 13)$ & 8 \\
BCSO11 & $-432(a 12-3 a 13+28 a 14)$ & 11 \\
FCSO11 & $432(\mathrm{a} 12+3 \mathrm{a} 13+28 \mathrm{a} 14)$ & 11 \\
CCSO12 & $1296(\mathrm{a} 13+28 \mathrm{a} 15)$ & 12 \\
\hline
\end{tabular}

\section{Conclusion}

In this paper, many high order schemes for approximating the first derivatives are constructed by solving the coefficients of a matrix system. By using Fourier analysis, the dispersion and dissipation errors of these schemes are calculated. When comparing dispersion and dissipation errors, the results show that high order schemes have fewer errors, which can be applied in computational problems with high frequency. In addition, the orders of the proposed schemes are verified by polynomial techniques. The upcoming project is to generalize this method to derive schemes of high order derivatives and apply these schemes in some CFD problems.

\section{Acknowledgment}

The authors would like to thank

Mustansiriyah university -

www.uomustansiriyah.edu.iq - (Baghdad - Iraq)

for its support in the present work.

\section{Author's declaration:}

- Conflicts of Interest: None.

- I hereby confirm that all the Figures and Tables in the manuscript are mine. Besides, the Figures and images, which are not mine, have been given the permission for re-publication attached with the manuscript.

- The author has signed an animal welfare statement.

- Ethical Clearance: The project was approved by the local ethical committee in University of Mustansiriyah.

\section{References}

1. Krishnan M, A Family of High Order Finite Difference Schemes with Good Spectral Resolution. J. Comput. Phys., 1998; 145(1):332-358.

2. Richard L B, Douglas F, Annette M B. Numerical Analysis. 10 ${ }^{\text {th }}$ Edition: Cengage Learning. 2016.

3. Sanjiva K L. Compact finite difference schemes with spectral-like resolution. J. Comput. Phys. 1992; 103(1):16-42.

4. Canuto C, Hussaini MY, Quarteroni, AM, Zang Th . Spectral Methods in Fluid Dynamics. SpringerVerlag, 1988.

5. Ford NJ, Morgado ML, Rebelo M. An implicit finite difference approximation for the solution of the diffusion equation with distributed order in time. ELECTRON T NUMER ANA. 2015;44:289-305.

6.Zhao-peng H, Zhi-zhong S, Wan-rong C. A FourthOrder Approximation of Fractional Derivatives with its Applications. J. Comput. Phys., 2015; 281(1):787805.

7. Sebastiano B, Francis F, Giovanni R. High Order Semi-Implicit Schemes for Time Dependent Partial Differential Equations. J. Sci. Comput. 2016; 68(3):975-1001.

8. Chi-Wang S, Stanley O. Efficient implementation of essentially non-oscillatory shock capturing schemes. J. Comput. Phys. 1989; 83(1):32-78.

9. Wang Z, Al-Dujaly HA, Liu C. Construction Methodology of Weighted Upwind Compact Scheme. In54th AIAA Aerospace Sciences Meeting 2016 (p. 2061).

10. Hassan A, Yong Y, Chaoqun L. Weighted Upwinding Compact Scheme for Shock Capturing, 55th AIAA Aerospace Sciences Meeting, AIAA SciTech Forum. 2017.

11. Wendt J F, Anderson JD. Computational fluid dynamics: The Basics with Applications. McGrawHill, International Edition. 1995; 236-237.

12. Hassan A, Talat A, Mustafa S, Saif H. IOP Conference Series Materials Science and Engineering IOP Conference Series Materials Science and Engineering 571:012035. August 2019. 


\section{صيغة المصفوفة لاشتقاق طرق عددية عالية الرتبة للمشتقة الأولى}

حسن عبل سلمان الاجيلي $\quad$ بينلين دونغ2

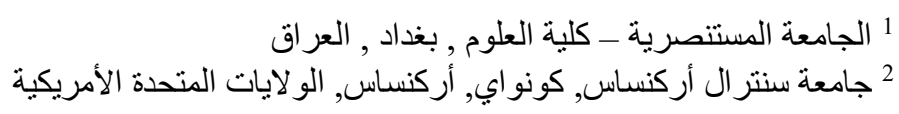

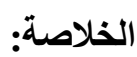

بالنسبة للعديد من المشكلات في الفيزياء وديناميكا الموائع الحسابية (CFD) ، فإن الحصول على تقريب دقيق للمشتقات يعد مهية

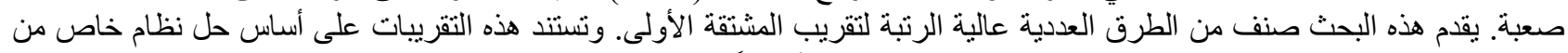

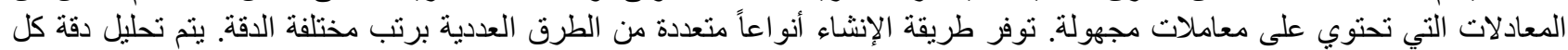

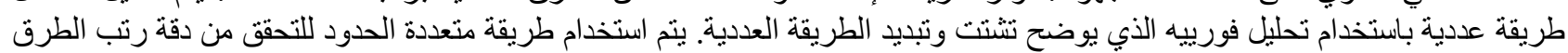

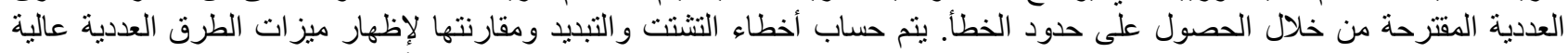

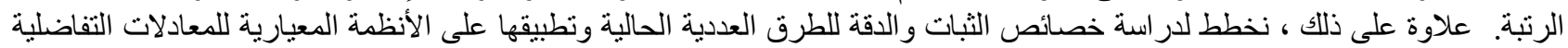

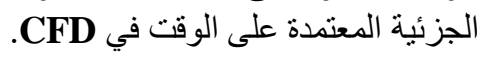
الكلمات المفتاحية: طرق عددية متر اصة ، نتتت ، تبديد ، رتبة عالية ، وحدة تردد الموجة 